\title{
Direkte Mikro- und Makroplastiktransportmessungen an großen und mittleren Flüssen sowie im Ablauf von Kläranlagen
}

\author{
Marcel Liedermann · Sebastian Pessenlehner · Philipp Gmeiner · Michael Tritthart · Philipp Hohenblum • \\ Gudrun Obersteiner · Helmut Habersack
}

Online publiziert: 10. August 2020

(C) Der/die Autor(en) 2020

Zusammenfassung Kunststoffabfälle als allgegenwärtige Verunreinigung unserer Umwelt geben aufgrund der weitgehend unbekannten langfristigen Auswirkungen auf Flora und Fauna zunehmend Anlass zur Sorge. Obwohl Flüsse als Transportwege von Kunststoffabfällen in Meere bekannt sind, konzentrierte sich die Forschung bisher vor allem auf das Vorkommen in den Weltmeeren. In den letzten Jahren wurden zwar einige Süßwasserstudien durchgeführt, aber kaum eine verwendete Methodik widmete sich der räumlichen Verteilung des Plastikmülls in der Wassersäule - insbesondere in Flüssen. Da Plastikpartikel aber nicht nur an der Oberfläche schwimmen, bedarf es zur Bestimmung des Transports unbedingt einer Methodik, die den Transport in verschiedenen Tiefen erheben kann und auch an mittleren und großen Flüssen anwendbar ist. Eine solche wurde in Österreich im Rahmen der Studie „Plastik in der Donau“ entwickelt und getestet. Die neue Methodik ermöglicht es, den Plas-

DI Dr. M. Liedermann $(\bowtie)$.

DI S. Pessenlehner, Bakk.techn. .

DI P. Gmeiner · PD DI Dr. M. Tritthart .

Univ.-Prof. DI Dr. H. Habersack

Department für Wasser - Atmosphäre

- Umwelt, Christian Doppler

Labor für Sedimentforschung

und -management, Institut

für Wasserbau, Hydraulik

und Fließgewässerforschung,

Universität für Bodenkultur Wien,

Muthgasse 107, 1190 Wien, Österreich

marcel.liedermann@boku.ac.at

DI P. Hohenblum

Umweltbundesamt, Spittelauer

Lände 5, 1090 Wien, Österreich

DI G. Obersteiner

Institut für Abfallwirtschaft,

Universität für Bodenkultur Wien,

Muthgasse 107, 1190 Wien, Österreich tiktransport in verschiedenen Tiefen entlang von Vertikalen zu messen, die innerhalb eines Profils verteilt sind. Das netzbasierte Gerät ist robust und kann auch bei hohen Fließgeschwindigkeiten und Abflüssen eingesetzt werden. Netze mit unterschiedlichen Größen $(41 \mu \mathrm{m}$, $250 \mu \mathrm{m}, 500 \mu \mathrm{m}$ und auch gröber für die Thematik Makroplastik) werden in drei verschiedenen Tiefen der Wassersäule positioniert. Im Auslaufbereich von Kläranlagen kann mit maßgeschneiderten Rahmen und Netzen relativ einfach der gesamte Abflussquerschnitt gefiltert und beprobt werden. Zur Untersuchung der Transportpfade und des Verhaltens einzelner Makroplastikpartikel, wurden selbige mit GPS-Sendern versehen, um ihre Positionen in hoher zeitlicher Auflösung zu verfolgen.

Der Einsatz des Netzmesssystems an der österreichischen Donau zeigte eine hohe Heterogenität der Mikroplastik-Konzentrationen innerhalb eines Messprofils. Aufgrund von turbulenter Durchmischung sowie unterschiedlicher Dichten der Polymere, der Aggregation und des Wachstums von Biofilmen kann der Kunststofftransport nicht auf die Oberflächenschicht eines Flusses beschränkt werden, sondern muss wie Schwebstoffe innerhalb der gesamten Wassersäule untersucht werden. Diese Ergebnisse implizieren, dass Vielpunktmessungen zur Ermittlung der räumlichen Verteilung der Kunststoffkonzentration unabdingbar sind und daher auch eine Voraussetzung für die Berechnung der Frachten darstellen. Darüber hinaus konnten an zwei Kläranlagen Messungen des Plastiktransports im Ablaufkanal durchgeführt werden. Es zeigte sich, dass die gewählte Methodik gut anwendbar ist und Restmengen an Mikrokunststoff gut entfernt werden könnten. An der Donau wurden erste Tests mit besenderten Makroplastikpartikeln durchgeführt, die ein hohes Potenzial für zukünftige Anwendungen im Forschungsbereich aufzeigen. So können die Transporteigenschaften der Partikel genau analysiert und auch Rückschlüsse über Ablagerung und Akkumulation von Plastikpartikeln gezogen werden.

Schlüsselwörter Mikroplastik . Makroplastik · Monitoring · Methodenentwicklung · Süßwasser

Direct micro- and macroplastic transport measurements on large and medium-sized rivers and in the outflow of wastewater treatment plants

Abstract Plastic waste as a persistent contamination of our environment is of increasing concern due to the largely unknown long-term effects on biota. Although rivers are known as transport paths for plastic waste into the sea, research has so far concentrated mainly on its occurrence in the sea. In recent years, there have been some freshwater studies, but hardly any methodology has been applied to the spatial distribution of plastic waste in the water column - especially in (large) rivers. However, since plastic particles do not only float on the surface, a methodology that addresses the transport at different depths and is also applicable to medium and large rivers is inevitable to determine the transport. Such a methodology has been developed and tested in Austria within the study "Plastics in the Danube". The new methodology enables the measurement of plastic transport at different depths of verticals distributed within a profile. The net-based device is robust and can be used even at high flow velocities and discharges. Nets of different sizes ( $41 \mu \mathrm{m}, 250 \mu \mathrm{m}, 500 \mu \mathrm{m}$ and also wider for macroplastics) are positioned at three different depths of the water column. In the outlet area of wastewater 
treatment plants, the entire discharge cross section can be filtered and sampled relatively simple with specifically built frames and nets. For questions concerning the transport paths and the behaviour of individual macroplastic particles, the particles were equipped with GPS transmitters in order to track their positions with high temporal resolution.

The use of the net measurement system at the Austrian Danube showed a high heterogeneity of the microplastic concentrations within a measurement profile. Due to turbulent mixing and because of different densities of polymers, aggregation and growth of biofilms, the transport of plastics cannot be limited to the surface layer of a river and has to be investigated like suspended solids within the whole water column. These results imply that multipoint measurements are indispensable to determine the spatial distribution of the plastic concentration and are therefore a prerequisite for the calculation of the loads. At two different wastewater treatment plants measurements of plastic transport in the discharge channel could be carried out. The chosen methodology is well applicable and shows that residual amounts of microplastics could be removed well. First tests with emitted macroplastic particles were carried out on the Danube and represent a high research potential. The transport properties of the particles can be analysed in detail and conclusions about the deposition and accumulation of plastic particles can be drawn.

Keywords Microplastics .

Macroplastics · Monitoring · Method development $\cdot$ Freshwater

\section{Einleitung}

Aufgrund ihrer hervorragenden Materialeigenschaften begannen Kunststoffe in den 1950er-Jahren ihren Siegeszug. Die weltweite Kunststoffproduktion steigt immer noch rapide an, von 230 Mio. Tonnen im Jahr 2005 auf 359 Mio. Tonnen im Jahr 2018 (Plastics Europe 2019). Somit werden jährlich etwa $40 \mathrm{~kg}$ Kunststoffe für jeden lebenden Menschen produziert. Zudem ist die Nutzungsdauer von Kunststoffen, insbesondere bei Einwegverpackungen, die etwa $40 \%$ des Kunststoffbedarfs in Europa ausmachen (Plastics Europe 2019), oft relativ kurz. Ihre primären
Vorteile, nämlich die Dichtheit und Widerstandsfähigkeit, sind gleichzeitig ihr Nachteil, da die Kunststoffe auch entsprechend resistent in unserer Umwelt verbleiben. Die Lebensdauer von Kunststoffen wird auf Hunderte bis Tausende von Jahren geschätzt, was bedeutet, dass abgesehen von der Verbrennung der überwiegende Teil aller jemals hergestellten Kunststoffe immer noch vorhanden ist (Barnes et al. 2009; Thompson et al. 2005). Obwohl die Recyclingraten über die letzten Jahre steigen (mit einer Rate von $64 \%$ im Zeitraum von 2006 bis 2014 in der EU28+2) (Plastics Europe 2019), geht ein Teil der kontrollierten Abfallströme verloren und landet in unserer Umwelt. Kunststoffabfälle werden nach ihrer Größe klassifiziert, es gibt allerdings keine definierte Größe für ein als „Mikroplastik“ kategorisiertes Partikel. Allerdings wird eine Obergrenze von $5 \mathrm{~mm}$ in der Literatur allgemein als vereinbart gesehen (Anderson et al. 2016; Cole et al. 2011). Plastikpartikel sind an Land und auch in der Süßwasserund Meeresumwelt präsent (z.B. Cole et al. 2011), wurden aber auch in den entlegensten Gebieten der Welt wie in Tiefseesedimenten (Van Cauwenberghe et al. 2013) oder eingekapselt im Eis der Arktis (Obbard et al. 2014) beobachtet. Wenn das Material dem Sonnenlicht, dem Wind und der chemischen Umgebung ausgesetzt wird, verändert es sich und zerfällt zu Teilchen von wenigen Mikrometern Größe, die leicht zerstreut werden können. Über die biologischen Auswirkungen, wie die Interaktion des Organismus mit Mikrokunststoffen und deren Aufnahme (Wagner et al. 2014), ist wenig bekannt, aber dass Partikel von Tieren mit Nahrung verwechselt werden, ist weithin nachgewiesen (Horton et al. 2017). Einem Team des österreichischen Umweltbundesamts ist es darüber hinaus gelungen, eine Methodik zu entwickeln, um das Vorhandensein von Plastik im menschlichen Stuhl zu überprüfen. In den Exkrementen aller Probanden wurde Mikroplastik nachgewiesen (Schwabl et al. 2019). Obwohl das Festland und Flüsse als Ursprung und Transportweg von Kunststoffen anerkannt sind, konzentriert sich der Großteil der bisherigen Forschung auf die Meeresumwelt (Horton et al. 2017). Studien an und in Flüssen sind jedoch in den letzten Jahren rasch vorangekommen. Horton et al. (2017) gaben einen detaillierten Überblick über Süß- wasserstudien, die von Seen (z. B. Imhof et al. 2016) bis zu Flüssen (z. B. Dris et al. 2015; Faure et al. 2015; Lechner et al. 2014; Mani et al. 2015) und Flusssedimenten reichen (z. B. Klein et al. 2015). Messungen wurden in den letzten Jahren in den Zuflüssen der Großen Seen (Baldwin et al. 2016), der Seine (Dris et al. 2015), verschiedener Flüsse in der Schweiz (Faure et al. 2015), des Rheins (Mani et al. 2015), und der Donau (Lechner et al. 2014) durchgeführt. Alle Forscher verwendeten benthische Netze (Lechner et al. 2014) oder Oberflächenschleppnetze (Mantatrawls), wie sie erstmals von Carpenter et al. (1972) verwendet, von Brown und Cheng (1981) beschrieben und von Lippiatt et al. (2013) als Standardmethode für Oberflächengewässer vorgeschlagen wurden. Bei dieser Methodik wird ein sogenannter „Manta-trawl“ horizontal an der Oberfläche durch das Wasser gezogen. Aufgrund dieser Probenahmestrategie befassen sich alle diese Studien nur mit den Partikeln, die auf der Wasseroberfläche und in der oberflächennahen Schicht (obere 10 bis $30 \mathrm{~cm}$ der Wassersäule) schwimmen. Moore et al. (2011) versuchten mehrere Tiefen $\mathrm{zu}$ erfassen, indem sie verschiedene Geräte, darunter einen modifizierten großen Helley-Smith-Probenehmer, in betonierten Bächen in der Nähe von Los Angeles einsetzten. Auch Dris et al. (2015) gingen auf verschiedene Tiefen in einem Punkt in der Mitte der Seine ein, indem sie ein Planktonnetz (das sogar Plastikfasern erfassen kann) mit einem Propellerströmungsmesser koppelten, um bis zu $2 \mathrm{~m}$ tief zu beproben. Bislang gab es jedoch keine anwendbare Methodik, um den gesamten Querschnitt mit einer Vielpunktmethode auch in der Tiefe standardmäßig zu beproben und so den Mikroplastiktransport in mittleren und großen Flüssen $\mathrm{zu}$ erfassen. Kunststoffe bestehen aus verschiedenen Polymeren, die je nach Zusammensetzung, Dichte und Form des Kunststoffs schwimmend, neutral oder sinkend sein können (Anderson et al. 2016; Cole et al. 2011). Sie können auch zu Polymeren höherer Dichte werden, wenn bei der Herstellung mineralische Füllstoffe hinzugefügt werden (Corcoran 2015). Kunststoffpartikel verändern ihre Größe und Dichte durch Aggregation oder durch das Wachstum von Biofilmen (z. B. Long et al. 2015; Harrison et al. 2018), und durch turbulente Strömung werden sie in Flüssen bewegt (Tritthart et al. 2019). Sie wer- 
den manchmal sogar in der Nähe des Flussbetts konzentriert und interagieren mit dem Sediment. In den Ozeanen sind in allen Schichten, von der Oberfläche bis hinunter zum Sediment, Plastikteilchen gefunden worden (z.B. Horton et al. 2017; Obbard et al. 2014; Dris et al. 2018). Wenn die Dichte des Kunststoffs geringer ist als die Dichte des Wassers, können sie schwimmen oder sich nahe der Wasseroberfläche befinden. Aufgrund ihrer unterschiedlichen Dichte, Turbulenzen in Flüssen, Biofilm usw. sind sie jedoch eher mit dem Verhalten von suspendierten Sedimentpartikeln $\mathrm{zu}$ vergleichen und kommen in der gesamten Wassersäule vor. Eine geeignete Methodik zur Bestimmung des Mikroplastiktransports sollte sich daher an den Forschungserfahrungen orientieren, die für Schwebstoffproben zur Verfügung stehen.

Dazu gibt es in Österreich einen Leitfaden, der die Messmethodik am Stand der Wissenschaft beschreibt und für die Verwendung im Land empfiehlt. Beim Schwebstofftransport wird in der Regel eine Kombination aus direkten und indirekten Methoden angewendet, um Messungen mit der erforderlichen räumlichen und zeitlichen Auflösung zu erfassen (Haimann et al. 2014). Um der zeitlichen Variabilität Rechnung $\mathrm{zu}$ tragen, werden optische Sensoren installiert, die die Trübung an einer Stelle im Gewässerquerschnitt kontinuierlich erfassen. Zusätzlich ist die Verteilung der Schwebstoffkonzentration im Querschnitt (räumliche Variabilität) $\mathrm{zu}$ berücksichtigen (Wass und Leeks 1999). Zur Ermittlung der mittleren Querschnittskonzentration $(\mathrm{Cm})$ wird die in ISO 4363 (2002) und von Edwards und Glysson (1999) beschriebene Mehrpunktmethode empfohlen. Mit dieser Methode werden die Schwebstoffkonzentration und die Fließgeschwindigkeit in verschiedenen vertikalen Profilen und in verschiedenen Tiefen gemessen (Haimann et al. 2014). Um die zeitliche Auflösung zu berücksichtigen, werden verschiedene Messungen über das ganze Jahr durchgeführt und $\mathrm{Ab}$ flussspektren oder indirekte Geräte verwendet. Angepasst an die Messung des Mikroplastiktransports ist es auch von hoher Bedeutung, die räumliche und zeitliche Variabilität zu bestimmen.

Neben der räumlichen Verteilung besteht die zweite wichtige Herausforderung bei Messungen in mittleren und großen natürlichen Fließgewässern darin, das Gerät an den erforder- lichen Stellen in der Wassersäule stabil $\mathrm{zu}$ halten. Hohe Strömungsgeschwindigkeiten und Turbulenzen sorgen für eine anspruchsvolle Umgebung, insbesondere bei der Handhabung großer Netze. Ähnliche Probleme treten bei Geschiebetransportmessungen auf und werden durch den Einsatz schwerer, hydraulisch optimierter Geräte gelöst. Liedermann et al. (2018a) haben dazu einen Korbprobenehmer (BfG-Probenehmer), der derzeit von der Bundesanstalt für Gewässerkunde in Koblenz, Deutschland, eingesetzt wird und auf dem Geschiebesammler von Delft Hydraulics (1958) basiert, adaptiert und für Geschiebetransportmessungen in der österreichischen Donau bis zu einem 200-jährlichen Hochwasserereignis $\left(10.738 \mathrm{~m}^{3} \mathrm{~s}^{-1}\right)$ eingesetzt (Liedermann et al. 2012, 2018a).

Die Maschenweiten variieren in den verschiedenen Studien (zwischen 80 und $800 \mu \mathrm{m})$, je nach Fokus der Arbeiten. In den meisten Studien in Flussumgebungen wurde eine Maschenweite von $300 \mu \mathrm{m}$ gewählt, um den Schwerpunkt auf primäre (in Mikrometergröße hergestellt) und sekundäre Mikrokunststoffe (Sekundärfragmente) $\mathrm{zu}$ legen. Bei der Beprobung von Fasern sollte ein noch kleinerer Durchmesser verwendet werden; so ermittelten Dris et al. (2018) beispielsweise eine 250-fach höhere Wahrscheinlichkeit, bei Verwendung eines 80-um-Maschennetzes Fasern zu entnehmen, als bei Verwendung eines 330- $\mu \mathrm{m}$-Maschennetzes.

Im Allgemeinen besteht eine Wissenslücke hinsichtlich des Vorhandenseins von Mikrokunststoffen in Süßwassersystemen (Obbard et al. 2014), und auch beim Prozessverständnis. Außerdem ist es von größter Bedeutung, die Methoden für die Sammlung, Verarbeitung und Analyse von Umweltproben zu standardisieren (Horton et al. 2017). Daher wurde versucht, eine Probenahmestrategie zu entwickeln, die auf mittlere und große Flüsse anwendbar ist und nicht nur die Wasseroberfläche, sondern den gesamten Transport von Kunststoffpartikeln in der Wassersäule berücksichtigt. Im Bereich der Erkenntnisse bezüglich der Herkunft und der Eintragspfade wurde eine Methodik für die Beprobung von Kläranlagen gesucht. Für das Prozessverständnis bezüglich der Transportpfade einzelner Plastikteile ist das Ziel, eine Methodik zur Verfolgung selbiger auf Basis von GPS-Sendern zu finden und erfolgreich anzuwenden.

\section{Methoden}

2.1 Messgerät zur Messung des Plastiktransports in mittleren und großen Fließgewässern

Zahlreiche Tests und Anpassungen waren notwendig, um eine passende und belastbare Konstruktion für das Messgerät zur Bestimmung des Plastiktransports in Fließgewässern zu entwickeln. Basis ist der rund $200 \mathrm{~kg}$ schwere Geschiebesammler, der als Geräteträger dient und dafür sorgt, dass die Netze gut absinken und in der vorgesehenen Tiefe positioniert werden können. Für die Extraktion des Plastiks stehen Netze in drei Tiefen zur Verfügung. Direkt an den Geräteträger angeheftet, wird mit einem Netz der Plastiktransport an der Gewässersohle abgedeckt. Die Einlauföffnung des bodennahen Netzes beträgt $300 \times 600 \mathrm{~mm}$ und kann mit Netzen unterschiedlicher Maschenweite bestückt werden. Für die oberflächennahe Beprobung wurde ein Stahlrahmen gestaltet, der Platz für zwei Netze mit $600 \times 600 \mathrm{~mm}$ Einlauföffnung bietet und weiters durch seitlich angebrachte Schwimmkörper gewährleistet, dass die Netze die Gewässeroberfläche beproben. Ein Rahmen, der nach Bestimmung der jeweiligen Wassertiefe und der Inklination des Seils durch einen Stopper auf die Mitte der Wassersäule eingestellt werden kann, bietet ebenfalls Platz für zwei Netze der Größe $600 \times 600 \mathrm{~mm}$. Das System bietet aber auch die Möglichkeit, die Methodik für den jeweiligen Anwendungsfall bzw. das jeweilig zu beprobende Fließgewässer anzupassen. So stehen mittlerweile auch schon Netze mit gröberen Maschenweiten und Rahmen mit Einlauföffnungsgrößen von $900 \times 900 \mathrm{~mm}$ zur Verfügung, um bei einer Fokussierung auf Makroplastik größere Kubaturen filtern zu können.

Die Rahmen sind mit einer langen Flosse ausgestattet, die eine ruhige Positionierung im Wasser gewährleisten und besitzen ein für die jeweilige Neigung des Seils einstellbares Gestell an der Front, damit die Rahmen immer aufrecht im Gewässer stehen. Jeder Rahmen kann im Zentrum der Netze mit einem mechanischen Flügel ausgestattet werden, über den die gefilterte Wassermenge berechnet werden kann. Am Ende der Netze ist jeweils ein Auffangbehälter angebracht, der nach der Spülung der Netze das Filtergut auffängt und relativ einfach entleert 


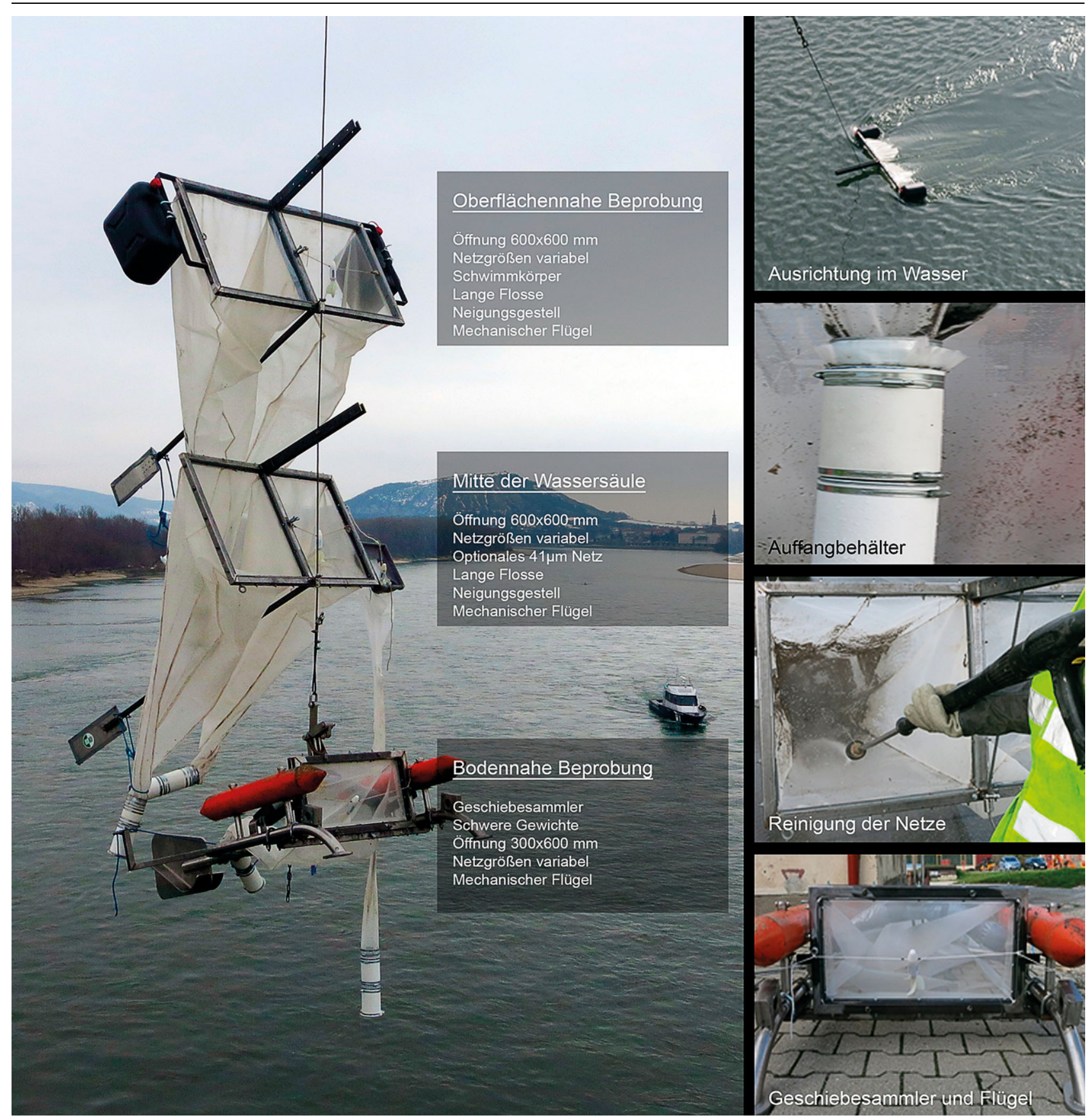

Abb. 1 Messgerät zur Beprobung des Plastiktransports in mittleren und großen Fließgewässern

werden kann. Die Netze sollten immer das erforderliche „offene Flächenverhältnis" von 3 (errechnet sich aus der Fläche der Einlauföffnung, der Gesamtfläche des Netztrichters und der Porosität des Netzstoffes) erreichen (Keen 2013); damit ist bei der 250-um- und bei der 500- $\mu \mathrm{m}$-Variante (die eine tatsächliche Porosität von $38 \%$ haben), eine Länge von etwa $2,5 \mathrm{~m}$ notwendig. Bei anderen Maschenweiten ist die Länge so zu wählen, dass wiederum ein of- fenes Flächenverhältnis von 3 erreicht werden kann. Das Messgerät und einige Details sind in Abb. 1 dargestellt.

An jedem Messprofil sollten 6 bis 7 Lotrechte über den gesamten benetzten Bereich verteilt werden, damit ergibt sich eine Einzelprobenzahl von ca. 35 Proben für eine Vielpunktmessung. Das Ziel sollte bei jeder Messung sein, die Netze so lange wie möglich auszubringen, da bei den Messungen ein maximales Wasservolumen gefil- tert werden sollte. Dennoch sollte eine Vielpunktmessung an einem Tag abgeschlossen sein, da konstante Bedingungen eine Voraussetzung für einen guten Datensatz sind. Daher wurde aufgrund der begrenzten Tageslichtzeit für die Messungen an der Donau die Probenahmedauer für jedes vertikale Profil auf 30 min festgelegt. Diese musste teilweise aus Zeitgründen (längere Reinigungszeiten) leicht reduziert oder bei Hochwasserereignismessun- 
gen aufgrund hoher Belastungen mit Schwebstoffen und organischer Substanz stark reduziert werden, um eine entsprechende Filterleistung sicherzustellen.

Zusätzlich zu dem mit dem mechanischen Durchflussmesser gemessenen Wasservolumen wurden ADCP-Messungen (Acoustic Doppler Current Profiler) durchgeführt, um die gesammelten Daten um die Strömungsverteilung und den Gesamtabfluss zu ergänzen.

Das gewonnene Material wird dann mit einem Hochdruckreiniger in den Probenbehälter gespült und in einen beschrifteten Behälter verbracht. Im Labor werden die Proben im Anschluss durch eine Siebkaskade gespült, um die Proben zu teilen und das weitere Vorgehen zu vereinfachen. Für die visuelle Sortierung werden den Proben Wasser und $\mathrm{NaCl}$ zugesetzt, um die Dichte der Flüssigkeit zu erhöhen und die Kunststoffpartikel aufschwimmen zu lassen. Bei Proben mit einem hohen Anteil an organischer Substanz kann $\mathrm{H}_{2} \mathrm{O}_{2}$ zugesetzt werden, da aber bei der Zersetzung größere organische Bestandteile (z. B. Blätter) nicht beziehungsweise nicht vollständig abgebaut wurden, ist diese Herangehensweise bei den meisten Proben aus Flüssen nicht vollständig zufriedenstellend. Nach der ersten Nasssortierung werden die Proben bei $50^{\circ} \mathrm{C}$ getrocknet und anschließend trocken nachsortiert. Die trockene organische Substanz kann dann leicht zermahlen werden, sodass Kunststoffteile besser sichtbar werden. Die Kunststoffpartikel werden in Fraktionen $<5 \mathrm{~mm}$ und $>5 \mathrm{~mm}$ getrennt und dann gewogen. Zur Identifizierung der Partikel wurde im Rahmen der Studie

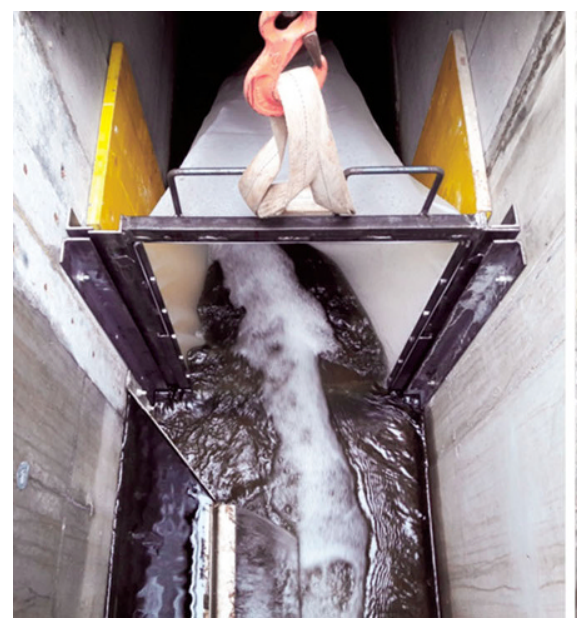

„Plastik in der Donau“ vom Umweltbundesamt ein ATR-IR (Attenuated Total Reflection Infrared Spectroscopy) verwendet (Hohenblum et al. 2015).

Die Plastikkonzentration $\mathrm{C}_{\mathrm{K}, \mathrm{i}, \mathrm{j}}\left[\mathrm{g} \mathrm{m}^{-3}\right]$ für jedes vertikale Profil (i) und jedes Netz (j) wird unter Verwendung der gemessenen Plastikmasse $m_{K, i, j}[g]$ und des gefilterten Wasservolumens $V_{i, j}\left[\mathrm{~m}^{3}\right]$ wie folgt berechnet:

$$
\mathrm{C}_{\mathrm{K}, \mathrm{i}, \mathrm{j}}=\mathrm{m}_{\mathrm{K} . \mathrm{i}, \mathrm{j}} / \mathrm{V}_{\mathrm{i}, \mathrm{j}}
$$

Vergleichbar mit der Analyse von Schwebstoffen in Flüssen (z. B. Haimann et al. 2014) kann dann der Plastiktransport für den Querschnitt und eine Abschätzung der Jahresfracht aus einer Reihe von Messungen bei unterschiedlichen Abflussbedingungen/ Randbedingungen ermittelt werden.

\subsection{Messgerät zur Beprobung des Plastiktransports im Ablaufkanal von Kläranlagen}

Zur Beantwortung der Fragestellung, wie viele Plastikpartikel eine Kläranlage nach allen Reinigungsstufen verlassen, wurde eine passende Methodik gesucht. Im Rahmen des Projekts „TEMPEST“ unter Koordination des Umweltbundesamts wird das Ziel verfolgt, Spurenstoffemissionen aus dem Kanalnetz zu erfassen. Ein Teilaspekt dabei sind auch Plastikpartikel mitsamt deren Herkunft und Transport in die Umwelt. Obwohl Kläranlagen Mikroplastik sehr effektiv zurückhalten, ist es speziell bei den kleinsten Fraktionen doch möglich, die einzelnen Klärstufen zu überwinden und in den Vorfluter zu gelangen. Um die tatsächliche Menge des ausgetrage- nen Materials gut erfassen zu können, ist es notwendig, den gesamten Querschnitt zu filtern und eine Netzweite zu wählen, die auf längere Zeitdauer nicht zu einem Rückstau im Netz führt. Es wurden zwei Kläranlagen im Osten Österreichs ausgewählt, die bereit waren, an einer derartigen testweisen Beprobung mitzuwirken. Anhand der dort vorherrschenden Randbedingungen wurden eine Methodik erarbeitet und mehrere Messungen durchgeführt.

Die verwendeten Messrahmen wurden aus Stahl gefertigt und so dimensioniert, dass sie den jeweiligen Kanal komplett abdecken. Seitlich im Kanal wurden Führungsschienen angebracht, die ein einfaches Absenken und Ziehen der Netze ermöglichen. Die verwendete Maschenweite der Netze betrug $500 \mu \mathrm{m}$. Die für die Messungen gewählten Stellen zur Entnahme der Proben wurden jeweils im Sammelkanal kurz vor der Einleitung in den Vorfluter gewählt. In der einen Kläranlage konnte damit ein Teilstrom, in der anderen das komplette geklärte Abwasser erfasst werden. Der genaue Messaufbau ist in Abb. 2 dargestellt.

Zusätzlich zu den Schienen wurden Holzvertäfelungen angeordnet, um ein Aufscheuern der Netze zu verhindern. Weiters wurde ein Verschluss für die Einlauföffnung aus Metall angefertigt, um - speziell bei Aufstau infolge einer Verlegung des Netzes - ein Rückfließen von Kunststoffpartikeln während des Ziehens des Messgeräts zu verhindern.
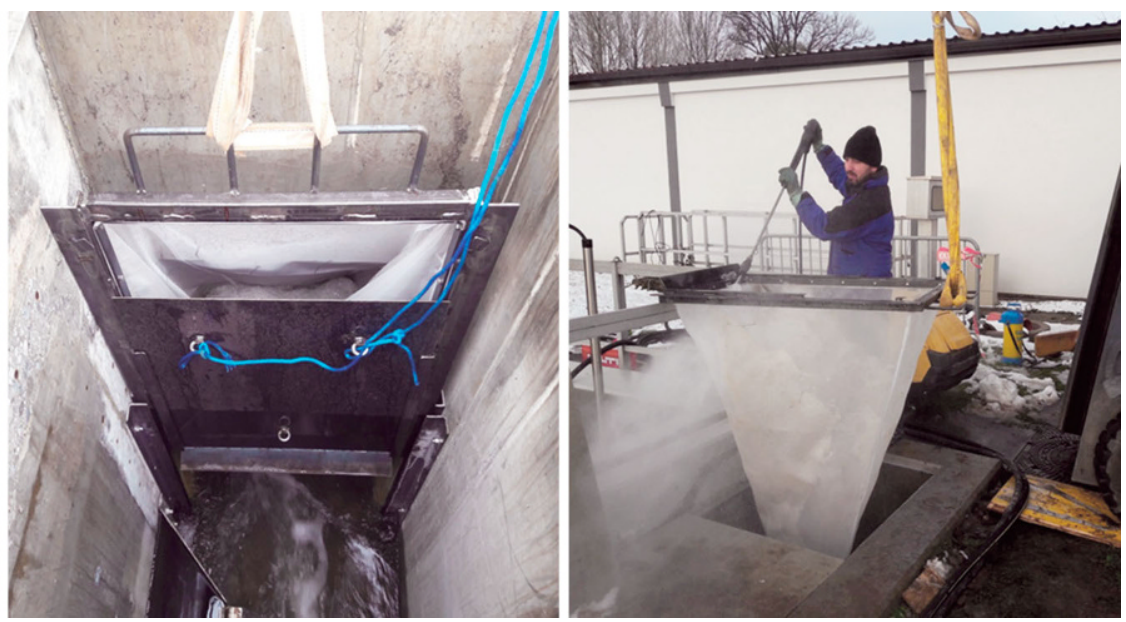

Abb. 2 Methodik zur Beprobung des Ablaufkanals von Kläranlagen 


\subsection{Besenderung von Plastikteilen mittels GPS-Technologie}

Speziell beim Thema Makroplastik stößt man bezüglich Quantifizierung und Prozessverständnis mit der reinen Beprobung des Transports mittels Netzen an Grenzen. Gerade in den österreichischen Flüssen ist aufgrund der großteils funktionierenden Abfalllogistik zwar Makroplastik zu finden, es liegt allerdings in einer Konzentration vor, bei der es innerhalb einer machbaren Messzeit schwer möglich ist, die $\mathrm{Zu}-$ fallskomponente bei der Beprobung $\mathrm{zu}$ eliminieren. Aktuell hat auch das Projekt „PlasticFreeDanube“ gezeigt, dass Makroplastikpartikel leicht und oft an wasserbaulichen Strukturen, Uferzonen und im Auenbereich anlanden und teilweise kurz, teilweise auch sehr lange verweilen. Hier wird es umso schwieriger, Transportprozesse $\mathrm{zu}$ verstehen und Gesetzmäßigkeiten abzuleiten, woher Partikel stammen und wohin sie weiter transportiert werden. Deshalb wird im Rahmen des Projekts „PlasticFreeDanube" aktuell an einer Methodik gearbeitet, Einzelpartikel unterschiedlicher Form und Beschaffenheit mit GPS-Sendern zu versehen und deren Transport in der Donau zu erfassen. Ähnlich wie schon bei Geschiebepartikeln an der österreichischen Donau angewandt (Liedermann et al. 2013) sollen so Einzelpartikel verfolgt und deren Pfade analysiert werden. Dazu wurde vorerst eine relativ kostenintensive, aber für den genannten Zweck schon in Frankreich erprobte Variante von GPSSendern angeschafft. Die Sender der
Firma INETIS haben den Vorteil, dass sie mit einer gut für derartige Zwecke abgestimmten Software geliefert werden, die es nicht nur erlaubt, die Senderpfade online $\mathrm{zu}$ speichern und $\mathrm{zu}$ analysieren, sondern auch viele Möglichkeiten der Programmierung bietet. So kann beispielsweise das Sendeintervall variabel adaptiert werden, um die Batterielaufzeit zu optimieren, womit relativ lange Laufzeiten erreicht werden können. Weiters kann verhindert werden, dass es bei schlechter Datenverbindung $\mathrm{zu}$ einer raschen Entleerung der Batterie kommt.

\section{Ergebnisse und Diskussion}

\subsection{Transportmessungen Donau}

Mit dem neu entwickelten Messgerät wurden zehn Vielpunktmessungen an zwei verschiedenen Messprofilen der österreichischen Donau durchgeführt (Aschach und Hainburg an der Donau), die unterschiedliche Abflussverhältnisse von Regulierungsniederwasser bis zu einem einjährlichen Hochwasserereignis abdeckten. Die Messungen zeigten deutlich, dass Kunststoff nicht nur in der obersten Schicht des Flusses zu finden ist, sondern über die gesamte Wassersäule verteilt ist (Abb. 3). Einige Messungen zeigten höhere Konzentration in Ufernähe beziehungsweise nur auf einer Seite des Flusses, aber bei anderen wurden die größten Konzentrationen in der Mitte des Flusses festgestellt.

Durch die Messung bei unterschiedlichen Durchflusssituationen an einer
Messstelle kann - ähnlich wie bei der Schwebstoffmessung - ein Zusammenhang zwischen Durchfluss und Plastiktransport hergestellt werden und über Durchflussganglinien Jahresfrachten ermittelt werden. Um diesbezüglich auf belastbare Ergebnisse zu kommen, müssen mehrere Messungen an den jeweiligen Messstellen durchgeführt werden. Erste Richtwerte wurden durch die bisherigen Messungen im Rahmen des Projekts „Plastik in der Donau“ ermittelt (Hohenblum et al. 2015). Der Plastiktransport ist stark vom Durchfluss der Donau abhängig. Bei Niederwasser ist der Transport in Aschach und Hainburg ähnlich (rund $3 \mathrm{~kg} / \mathrm{d}$ bei beiden Messstellen), bei höheren Durchflüssen wird in Hainburg deutlich mehr Plastik gemessen. Der durchschnittliche Plastiktransport liegt in Aschach zwischen 10 und $59 \mathrm{~kg}$ pro Tag und in Hainburg bei 7 bis $161 \mathrm{~kg}$ pro Tag. Mithilfe der Durchfluss-Jahresganglinien der letzten Jahre konnte daraus die Plastik-Fracht abgeschätzt werden. Für die beprobten Fraktionen (Makroplastik war nicht Ziel der Studie) wurde die Jahresfracht in Aschach mit $<14$ Tonnen pro Jahr, in Hainburg mit $<41$ Tonnen pro Jahr, abgeschätzt.

\subsection{Messung Auslaufkanäle Kläranlagen}

Durch die verwendete Methodik an den Auslaufkanälen der Kläranlagen konnte das gesamte Ablaufvolumen über jeweils 4-mal eine Stunde je Probentag gefiltert werden. Durch die grundsätzlich geringe Belastung des Wassers mit

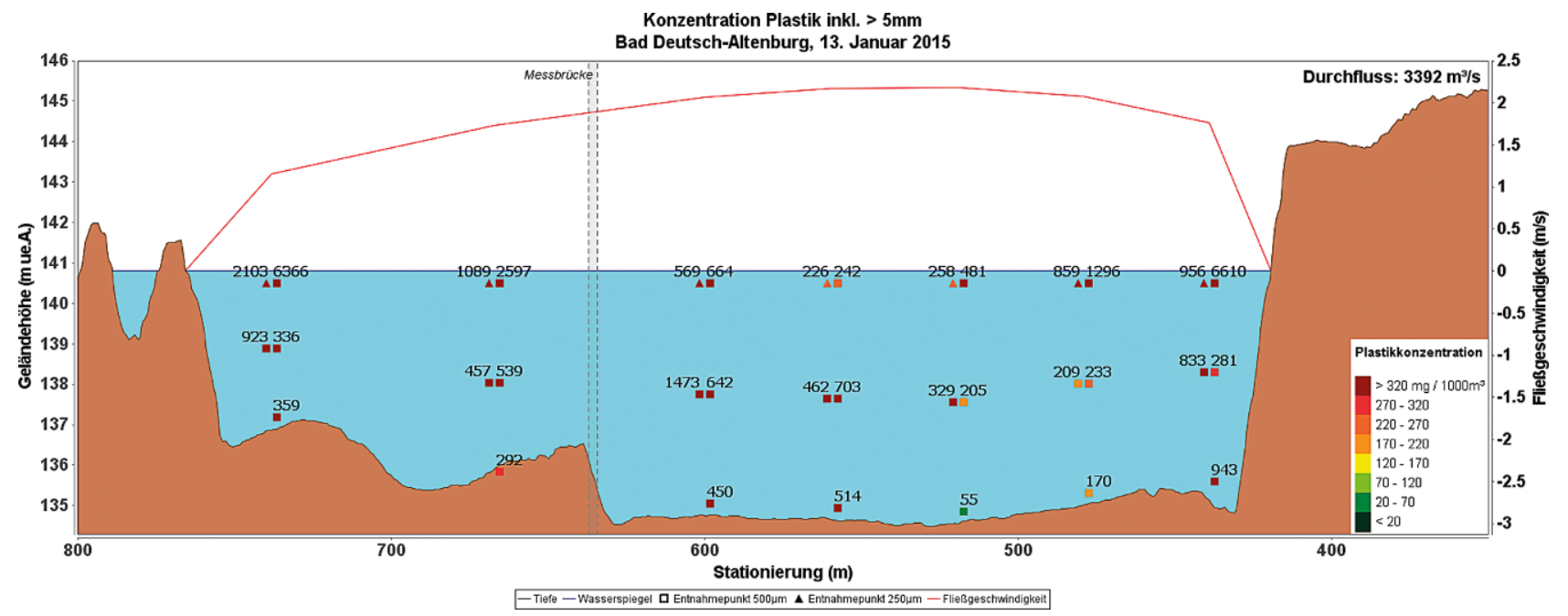

Abb. 3 Darstellung des Ergebnisses einer Vielpunktmessung des Plastiktransports bei Hainburg (adaptiert nach Liedermann et al. 2018b) 

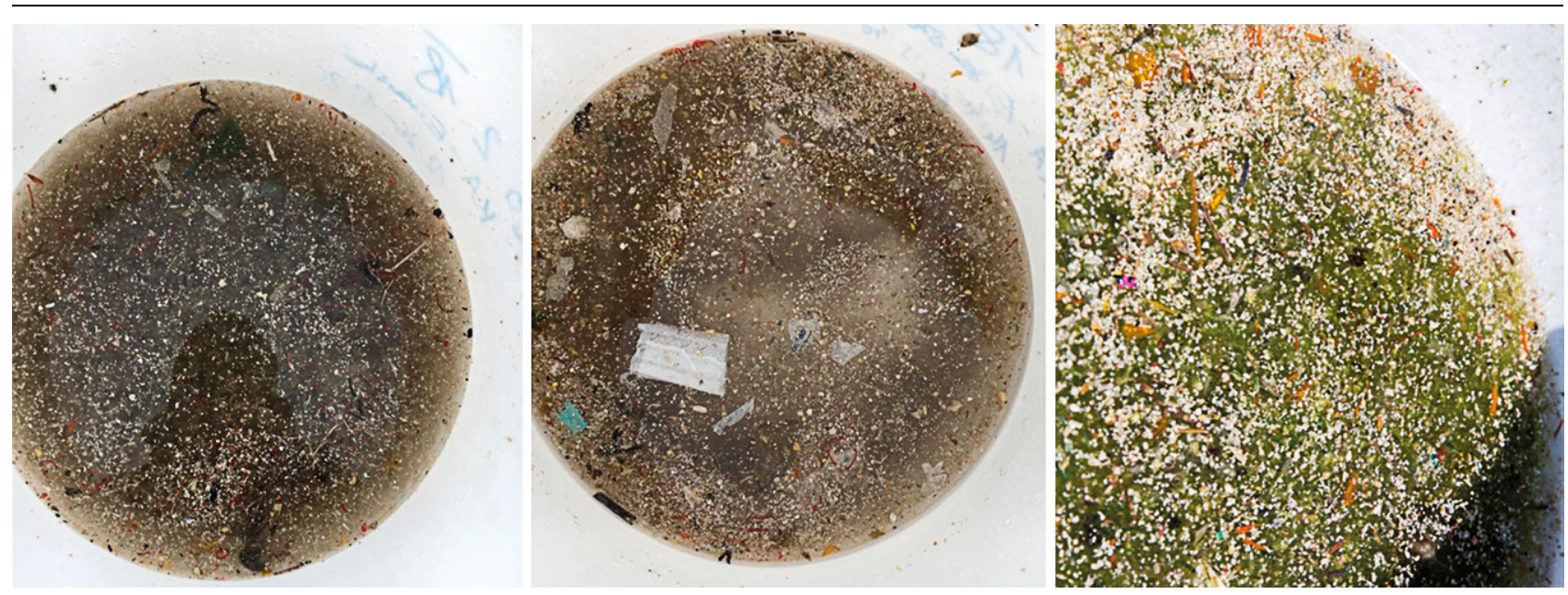

Abb. 4 Ausgespültes, gefiltertes Material im Probenbehältnis

Schweb- und Schwimmstoffen wären auch deutlich höhere Mess- bzw. Filterzeiten ohne Verlegen des Netzes möglich. Das Sperren des gesamten Querschnitts ermöglicht es, die Probe einem gut definierten Wasservolumen zuzuordnen. So konnten bis zu $2500 \mathrm{~m}^{3}$ Ablaufwasser in den 4 Messstunden gefiltert werden. Fotos des ausgespülten Filtermaterials sind in Abb. 4 zu sehen. Die Proben werden im Rahmen des Projekts „TEMPEST“ an der TU Wien und am Umweltbundesamt mit verschiedenen Methoden analysiert, um diesbezüglich eine effektive Vorgehensweise zu entwickeln.

Die visuelle Analyse zeigt aber bereits, dass zwar verhältnismäßig wenig Plastik die Reinigungsstufen der Klär-
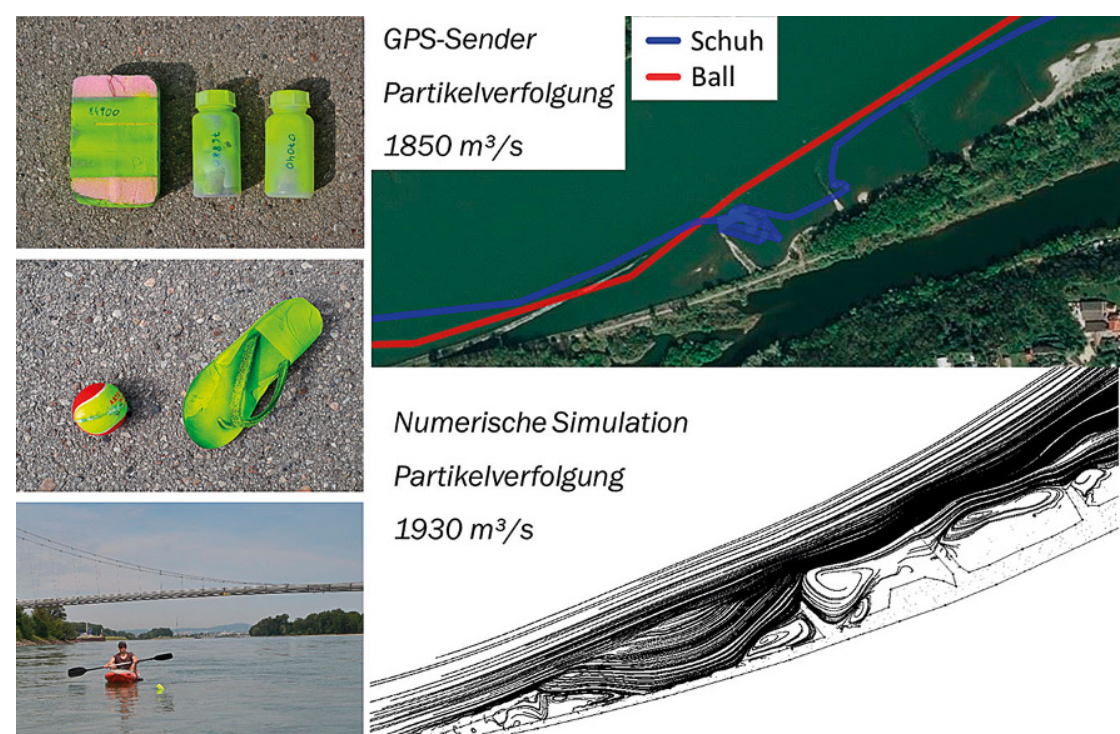

Abb. 5 Erste Tests und Ergebnisse der GPS-Tracer: Verwendete Kunststoffpartikel und Begleitung der Tracer (links), Vergleich beobachtete Partikelpfade (rechts, oben) anlagen passiert, aber doch speziell Mikroplastik in den Proben zu finden ist. Aufgrund der relativ einfachen und wartungsarmen Installationsmöglichkeiten eines Filters im Ablaufkanal könnten die Restmengen - zumindest bis zu einer gewissen Größe - relativ leicht aus dem geklärten Wasser entfernt werden.

\subsection{GPS-Tracing an der Donau}

Bezüglich der GPS-Tracer wurden erste Tests an der Donau durchgeführt. Dazu wurden unterschiedliche Makroplastikteile, die im Rahmen des Projekts „PlasticFreeDanube“ häufig im Nationalpark Donau-Auen gefunden werden (PET-Flaschen, PU-Schaum, Schuhe, Tennisbälle etc.), mit den Senund modellierte Partikelpfade (rechts, unten)



Numerische Simulatio

Partikelverfolgung

$1930 \mathrm{~m}^{3} / \mathrm{s}$ dern bestückt und vorerst mit Kanus begleitet. Die Partikel wurden im Donaukanal freigesetzt, um den Weg eines Partikels mit diesem Eintragspfad dokumentieren zu können. Die Partikel konnten gut verfolgt bzw. begleitet werden und die Aufzeichnung der Pfade funktionierte einwandfrei. Die Partikel strandeten häufig in Abschnitten, die im Projekt durch Begehungen sowie Ufer- und Hinterlandsammlungen als Akkumulationszonen definiert wurden. Die zurückgelegten Pfade konnten zur Validierung des im Projekt aufgebauten hydrodynamisch-numerischen Modells herangezogen werden. Das Modell ermöglicht es, virtuelle Partikel im modellierten System zu starten und $\mathrm{zu}$ verfolgen. Abb. 5 zeigt die verwendeten Artikel sowie erste Ergebnisse der Partikelverfolgung im Feld sowie die Simulation virtueller Plastikpartikel im hydrodynamisch-numerischen Modell unter Mittelwasserverhältnissen im gleichen Gewässerabschnitt der Donau.

\section{Schlussfolgerungen und Ausblick}

Ein neues Gerät wurde vorgestellt, das sich erstmals mit dem Transport von Mikrokunststoffen in verschiedenen Tiefen und mehreren vertikalen Profilen in mittleren und großen Flüssen befasst. Es ist robust und auch bei hohen Fließgeschwindigkeiten und Abflüssen einsetzbar. Erste Messungen haben gezeigt, dass Vielpunktmessungen notwendig sind, um die räumliche Verteilung der Kunststoffkonzentration $\mathrm{zu}$ erfassen und somit eine Voraussetzung für die Berechnung des stattfindenden Transports darstellen. Auf Basis der Erkenntnisse wird vorgeschlagen, 
dass diese Methodik als Standard dienen soll, um den Mikroplastiktransport in Flüssen zu untersuchen.

Die Beprobung der Auslaufbereiche zweier Kläranlagen im Osten Österreichs hat gezeigt, dass es mit einer relativ einfachen Methodik möglich ist, den gesamten Volumenstrom bis $\mathrm{zu}$ einer Größe von $500 \mu \mathrm{m}$ zu filtern. Die visuelle Analyse lässt vermuten, dass in den Klärstufen relativ viel Plastik extrahiert werden kann; trotzdem könnte eine nennenswerte Menge an Mikroplastik durch mechanische Filterung zusätzlich entfernt werden und würde damit nicht in die Vorflut und somit in unsere Umwelt gelangen.

GPS-Tracer wurden zur Verfolgung von Makroplastikpartikeln verwendet und stellen eine vielversprechende Methodik dar, um mehr über die Herkunft und das Verhalten derartiger Partikel im Fließgewässer zu erfahren. Die Ergebnisse können weiters zur Kalibrierung und Validierung computergestützter Modelle verwendet werden und damit auch weitreichendere Informationen für Zustände und Randbedingungen liefern, die nicht beprobt werden können.
Danksagung Die Studie „Plastik in der Donau" wurde vom Bundesministerium für Landwirtschaft, Regionen und Tourismus BMLRT (vormals BMNT) sowie von den Landesregierungen von Niederösterreich, Oberösterreich und Wien finanziert. Das Projekt wurde vom Umweltbundesamt Österreich koordiniert und mithilfe von viadonau umgesetzt. Das Projekt „PlasticFreeDanube“ wird durch die Europäische Union im Rahmen des INTERREG V-A SlovakiaAustria 2014-2020 (ERDF)-Programms finanziert. Das Projekt „TEMPEST“ wird vom Umweltbundesamt koordiniert und vom BMLRT (vormals BMNT) unterstützt. Weiters danken wir für die finanzielle Unterstützung durch das Bundesministerium für Digitalisierung und Wirtschaftsstandort und die Nationalstiftung für Forschung, Technologie und Entwicklung. Dank gebührt außerdem Martin Hinterleitner, Lukas Sölkner und Lukas Knafl für die Mithilfe bei den Freilandarbeiten.

Funding Open access funding provided by University of Natural Resources and Life Sciences Vienna (BOKU).
Open Access Dieser Artikel wird unter der Creative Commons Namensnennung 4.0 International Lizenz veröffentlicht, welche die Nutzung, Vervielfältigung, Bearbeitung, Verbreitung und Wiedergabe in jeglichem Medium und Format erlaubt, sofern Sie den/die ursprünglichen Autor(en) und die Quelle ordnungsgemäß nennen, einen Link zur Creative Commons Lizenz beifügen und angeben, ob Änderungen vorgenommen wurden.

Die in diesem Artikel enthaltenen Bilder und sonstiges Drittmaterial unterliegen ebenfalls der genannten Creative Commons Lizenz, sofern sich aus der Abbildungslegende nichts anderes ergibt. Sofern das betreffende Material nicht unter der genannten Creative Commons Lizenz steht und die betreffende Handlung nicht nach gesetzlichen Vorschriften erlaubt ist, ist für die oben aufgeführten Weiterverwendungen des Materials die Einwilligung des jeweiligen Rechteinhabers einzuholen.

Weitere Details zur Lizenz entnehmen Sie bitte der Lizenzinformation auf http://creativecommons.org/licenses/ by/4.0/deed.de.
Anderson, J.C., Park, B.J., Palace, V.P. (2016): Microplastics in aquatic environments: Implications for Canadian ecosystems. Env. Poll., 218, 269-280.

Baldwin, A.K., Corsi, S.R., Mason, S.A. (2016) Plastic debris in 29 Great Lakes tributaries: relations to watershed attributes and hydrology. Environ. Sci. Technol., 50, 10377-10385.

Barnes, D.K., Galgani, F., Thompson, R.C., Barlaz, M. (2009): Accumulation and fragmentation of plastic debris in global environments. Philos.

Trans. R. Soc.B, 364, 1985-1998.

Brown, D.M., Cheng L. (1981): New net for sampling the ocean surface, Marine Ecology Progress Series, 5, 225-227.

Carpenter, E.J., Anderson, S.J., Harvey G.R. Miklas H.P., Peck, B.B. (1972): Polystyrene Spherules in Coastal Waters. Science, 178(4062), 749-750.

Cole, M., Lindeque, P., Halsband, C., Galloway,

TS. (2011): Microplastics as contaminants in the marine environment: a review. Mar. Pollut. Bull. $62,2588-2597$

Corcoran, P.L. (2015): Benthic plastic debris in marine and fresh water environments. Environ. Sci.: Processes Impacts, 17, 1363-1369.

Delft Hydraulics (1958): Calibration of BTMA (in Dutch). Report M6Q1I. Delft Hydraulics Laboratory: Delft.

Dris, R., Gasperi, J., Rocher, V., Saad, M., Renault, N., Tassin, B. (2015): Microplastic contamination in an urban area: a case study in Greater Paris. Environ. Chem., 12, 592.

Dris, R., Gasperi, J., Rocher, V., Tassin, B. (2018): Synthetic and non-synthetic anthropogenic fibers in a river under the impact of Paris Mega- city: Sampling methodological aspects and flux estimations, Science of The Total Environment, 618, 157-164.

Edwards, T., Glysson, G. (1999): Field methods for measurement of fluvial sediment. In Techniques of Water-Resources Investigations of the U.S. Geological Survey, Book 3, Application of Hydraulics; United States Government Printing Office: Washington, DC, USA; Chapter C2; p. 89 Faure, F., Demars, C., Wieser, O., Kunz, M., de Alencastro, L.F. (2015): Plastic pollution in Swiss surface waters: nature and concentrations, interaction with pollutants. Environ. Chem., 12, 582. Haimann, M., Liedermann, M., Lalk, P., Habersack, H. (2014): An integrated suspended sediment transport monitoring and analysis concept Int. Journal of Sediment Research 2014 29, 135-148.

Harrison, J., Hoellein, T., Sapp, M., Tagg, A. Ju-Nam, Y., Ojeda, J. (2018): Microplastic-Associated Biofilms: A Comparison of Freshwate and Marine Environments. Martin Wagner, Scott Lambert (Ed.), Freshwater Microplastics, Springer, Cham. https://doi.org/10.1007/978-3-31961615-5_9

Hohenblum, $\mathrm{P}$, Frischenschlager, $\mathrm{H}_{\text {, }}$ Reisinger, H., Konecny, R., Uhl, M., Mühlegger, S., Habersack, H., Liedermann, M., Gmeiner, P., Weidenhiller, B., Fischer, N., Rindler, R. (2015): Plastik in der Donau - Untersuchung zum Vorkommen von Kunststoffen in der Donau in Österreich; Report Umweltbundesamt; REP-0547.

Horton, A.A., Walton, A., Spurgeon, D.J., Lahive, E., Svendsen, C. (2017): Microplastics in freshwater and terrestrial environments: Evaluating the current understanding to identify the know- ledge gaps and future research priorities. Science of The Total Environment, 586, 127-141.

Imhof, H.K., Laforsch, C., Wiesheu, A.C., Schmid, J., Anger, P.M., Niessner, R., Ivleva, N.P. (2016): Pigments and plastic in limnetic ecosystems: a qualitative and quantitative study on microparticles of different size classes. Water Res., 98, 64-74

ISO 4363 (2002): Measurement of liquid flow in open channels-Methods for measurement of characteristics of suspended sediment. International standard, Geneva, Switzerland.

Keen, E.; (2013): A practical designer's guide to mesozooplankton nets. Available: http:// acsweb.ucsd.edu/ ekeen/resources/Choosinga-Net.pdf. Zugegriffen: 05/2018

Klein, S., Worch, E., Knepper, T.P. (2015): Occurrence and spatial distribution of microplastics in river shore sediments of the rhine-main area in Germany. Environ. Sci. Technol., 49, 6070-6076.

Lechner, A., Keckeis, H., Lumesberger-Loisl, E, Zens, B., Krusch, R, Tritthart, M., Glas, M. Schludermann, E. (2014): The Danube so colourful: a potpourri of plastic litter outnumbers fish larvae in Europe's second largest river. Environ. Pollut., 188, 177-181.

Liedermann, M., Gmeiner, P., Kreisler, A., Tritthart, M., Habersack, H. (2018a): Insights into bedload transport processes of a large regulated gravel-bed river. Earth Surf. Process. Landforms, 43, 514-523.

Liedermann, M., Gmeiner, P., Niederreiter, R., Tritthart, M., Habersack, H. (2012): Innovative Methoden zum Geschiebemonitoring am Beispiel der Donau Österreichische Wasser- und Abfallwirtschaft, 64, 527-534. 


\section{Originalarbeit}

Liedermann, M., Tritthart, M., Habersack, H. (2013): Particle path characteristics at the large gravel-bed river Danube: results from a tracer study and numerical modelling. Earth Surface Processes and Landforms, 38, 512-522.

Liedermann, M; Gmeiner, P; Pessenlehner, S; Haimann, M; Hohenblum, P; Habersack, $H$. (2018b): A Methodology for Measuring Microplastic Transport in Large or Medium Rivers Water 2018, 10(4), 414; https://doi.org/10.3390/ w10040414

Lippiatt, S., Opfer, S., Arthur,C. (2013): Marine debris monitoring and assessment: Recommendation for monitoring debris trends in the marine environment; NOAA Technical Memorandum NOS-OR\&R-46, 2013.

Long, M., Moriceau, B., Gallinari, M., Lambert, C., Huvet, A., Raffray, J., Soudant, P. (2015): Interactions between microplastics and phytoplankton aggregates: impact on their respective fates. Mar. Chem., 175, 39-46.

Mani, T., Hauk, A., Walter, U., Burkhardt-Holm, P. (2015): Microplastics profile along the Rhine River. Sci. Rep. 5, 17988.

Moore, C.J., Lattin, G.L., Zellers, A.F. (2011): Quantity and type of plastic debris flowing from two urban rivers to coastal waters and beaches of Southern California. Journal of Integrated Coastal Zone Management, 11, 65-73.

Obbard, R.W., Sadri, S., Wong, Y.Q., Khitun, A.A., Baker, I., Thompson, R.C. (2014): Global warming releases microplastic legacy frozen in Arctic Sea ice. Earth's Future, 2, 315-320.

PlasticsEurope (2019): Plastics-the Facts 2019, An Analysis of European Plastics Production, Demand and Waste Data. Plastics Europe, Association of Plastic Manufacturers, Brussels.

Schwabl, P., Köppel, S., Königshofer, P., Bucsics, T., Trauner, M., Reiberger, T., Liebmann, B. (2019): Detection of Various Microplastics in Human Stool: A Prospective Case Series. Ann Intern Med., 171, 453-457.

Thompson, R.C., Moore, C., Andrady, A., Gregory, M., Takada, H., Weisberg, S. (2005): New directions in plastic debris. Science 310, 1117.

Tritthart, M., Gmeiner, P., Liedermann, M. Habersack, H. (2019): A meso-scale gravel tracer model for large gravel-bed rivers. Journal of Applied Water Engineering and Research, 7, 89-102.

Van Cauwenberghe, L., Vanreusel, A., Mees, J., Janssen, C.R. (2013): Microplastic polluti- on in deep-sea sediments. Environ. Pollut.,182, 495-499.

Wagner, M., Scherer, C., Alvarez-Muñoz, D., Brennholt, N., Bourrain, X., Buchinger, S., Fries, E., Grosbois, C., Klasmeier, J., Marti, T., Rodriguez-Mozaz, S., Urbatzka, R., Vethaak, A.D., Winther-Nielsen, M., Reifferscheid, G. (2014): Microplastics in freshwater ecosystems: what we know and what we need to know. Environ. Sci. Eur. https://doi.org/10.1186/s12302014-0012-7

Wass P.D., Leeks, J.L. (1999): Suspended sediment fluxes in the Humber catchment, UK. Hydrological Processes, 13, 935-953.

Hinweis des Verlags Der Verlag bleibt in Hinblick auf geografische Zuordnungen und Gebietsbezeichnungen in veröffentlichten Karten und Institutsadressen neutral. 